\title{
Editorial: Compelling COVID-19 Graphical Simulations
}

\author{
Mina C. Johnson-Glenberg * \\ Arizona State University, Tempe, AZ, United States
}

Keywords: data visualization, emerging technologies, COVID-19, simulations, games for health

\author{
Editorial on the Research Topic
}

\section{Compelling COVID-19 Graphical Simulations}

When COVID-19 was declared a pandemic in the early spring of 2020, thousands of research labs and offices around the world stopped business as usual and started putting efforts into understanding how the COVID-19 virus spread. We assumed that some groups would also put efforts into creating models, or simulations, to help share knowledge with the public. The goal of this collection of "compelling sims" was to create a historical snapshot of some of the visualizations and simulations that were built out by the end of 2020. The three editors suspected that innovative new methods for relaying complex information to the public might emerge and this collection of articles from 11 different countries, seven businesses, and multiple universities from around the world shows how diverse the community answering this call was. The series focuses on new tools designed to aid our understanding of COVID-19 and some new methods for disseminating information for public health. The editors purposefully kept the format type of the articles flexible. The goals were to encourage the inclusion of multimedia elements (e.g., links showcasing the simulations or platforms), and to meet the tight deadline of submission by the end of 2020. Thus, we did not insist on formal experimental verification studies. Because this field is moving quickly, we were also interested in the design aspects of these simulations and/or platforms.

We hope to learn from each other as we all continue to create simulations and interactive data platforms. Sometimes the design perspective is highlighted as game designers pivoted from interactive AR to the more accessible PC platform (see Interactive CovidCampus). Sometimes interdisciplinary and international projects are highlighted like the CoronaSurveys System designed to infer the incidence of COVID-19 around the world using anonymous open surveys. Sometimes the flexibility and interactivity of the technology is highlighted like the mixed reality tool where groups of people can interact in real-time with the latest COVID-19 statistics in a virtual space on multiple platforms (e.g., smart phone, tablet, PC, or VR headset) using Flow. This series shows evidence that research and business communities stepped up and created a variety of tools and sims to move forward our understanding of the pandemic. The editors would like to thank the authors and reviewers for all their diligent work. Below is a short description of each article: (Listed by number of views in September 2020).

Received: 19 September 2021 Accepted: 22 September 2021 Published: 19 October 2021

\section{- The CoronaSurveys System for COVID-19 Incidence Data Collection and Processing}

CoronaSurveys is an ongoing interdisciplinary project designed to develop a system to infer the incidence of COVID-19 around the world using anonymous open surveys. The surveys have been translated into 60 languages and are continuously collecting participants' responses. Several architecture and system design challenges are discussed. 
- Interactive CovidCampus Simulation Game: Genesis, Design, and Outcomes

The CovidCampus game experience was designed to help college students understand how their decisions can affect their probability of infection throughout a day on campus. Eight decision points were created and a probability of infection bar/icon kept score at the bottom of the screen. Within group comparisons of immediate learning gains and self-reported behavioral changes revealed a significant increase in confidence in asking safety-related questions after playing the game. A significant majority of players listed new actions they would take to increase their safety. Additionally, players were more agentic in their choices. The browser-based game can be played on a computer. These results suggest that even short, interactive desktop games may be effective methods for disseminating information about how to stay safer during a pandemic.

- Flow Immersive: A Multiuser, Multidimensional, Multiplatform Interactive Covid-19 Data Visualization Tool

This article explores the design and usability of a data storytelling platform called Flow Immersive. The interactive tool can be used with multiple platforms including smart phones, tablets, PCs, and AR or VR headsets. It was designed to engage both laypeople and experts in understanding complex visual information. This tool integrates emerging technologies (e. g., augmented reality (AR) and virtual reality (VR)) with a multiplatform, multiuser publishing approach.

- Visual Continuity of Protein Secondary Structure Rendering: Application to SARS-CoV-2 Mpro in Virtual Reality

Ribbon diagrams are important for protein visualization. The SARS-CoV-2 main protease has been imaged in a clear and concise manner, but most imagining algorithms do not maintain visual continuity when viewing a molecular trajectory. This team outlines a new method which prevents visual "ribbon flipping" discontinuities; those can be visually distracting when they morph between consecutive cross sections instead of rotating. This article illustrates the utility of a new, aesthetically more pleasing, algorithm to visualize iMD-VR (interactive molecular dynamics in virtual reality) simulations of the secondary structure of the SARS-CoV-2 main protease.

- Simulation Based on an Agent-based Model to Demonstrate the Transmission of COVID-19 and Effectiveness of Different Public Health Strategies

The team created an agent-based model (ABM) to simulate the transmission of the COVID-19 virus in the real world and to demonstrate several methods for mitigating its spread. As the player, you have control over two powerful public health strategies including masking and stay-at-home rules. The game simulation can be played using links in the article.

\section{AUTHOR CONTRIBUTIONS}

The author confirms being the sole contributor of this work and has approved it for publication.

Conflict of Interest: The author declares that the research was conducted in the absence of any commercial or financial relationships that could be construed as a potential conflict of interest.

Publisher's Note: All claims expressed in this article are solely those of the authors and do not necessarily represent those of their affiliated organizations, or those of the publisher, the editors and the reviewers. Any product that may be evaluated in this article, or claim that may be made by its manufacturer, is not guaranteed or endorsed by the publisher.

Copyright (c) 2021 Johnson-Glenberg. This is an open-access article distributed under the terms of the Creative Commons Attribution License (CC BY). The use, distribution or reproduction in other forums is permitted, provided the original author(s) and the copyright owner(s) are credited and that the original publication in this journal is cited, in accordance with accepted academic practice. No use, distribution or reproduction is permitted which does not comply with these terms. 